when Aerobacter aerogenes was growing at a rate of $0.1 \mathrm{~h}^{-1}$ and an even more pronounced (seventeen-fold) increase when the growth rate was increased to $0.3 \mathrm{~h}^{-1}$. These changes in pool size resulted very largely from disproportionate increases in glutamate. The response of $A$. aerogenes to salt was rapid and reflected a de novo synthesis of glutamate. Although other Gram negative bacteria showed a similar rapid response to $\mathrm{NaCl}$, Gram positive species showed an enhancement of the free amino-acid pool only after prolonged exposure to $\mathrm{NaCl}$.

The full interpretation of these data remains to be made but some fascinating control systems are indicated. The rapid change in glutamate concentrations in Gram negative bacteria is most likely caused by the activity of altered glutamate dehydrogenase. In Aerobacter, Tempest and his colleagues found that this enzyme showed marked $p \mathrm{H}$ dependence and, arguing that addition of salt would cause a rapid efflux of protons and hence an increase in the intracellular $p \mathrm{H}$, they suggest that this is the most feasible explanation for the rapid increase in glutamate. Subsequently the intracellular proton concentration would be restored and glutamate synthesis established at a new steady state rate. Whereas the maintenance of a constant proton concentration in Gram negative bacteria may control the activity of glutamate dehydrogenase and hence the level of free glutamate, Tempest and his colleagues (ibid., 187) have found that similar reasoning does not explain the notable difference in glutamate concentrations of Gram negative and Gram positive type.

The amino-acid pools of the yeast Saccharomyces cereviseae were not affected either quantitatively or qualitatively by sodium chloride; rather the supply of ammonium ions was the controlling factor. Nevertheless, the recent work of Watson (ibid., 91) indicates at least one important way in which salt influences yeast growth. Using salt concentrations higher than those used by Tempest et al., Watson has shown that the maximum specific growth rate of yeast falls when sodium. chloride is added to the growth medium. Studies of steady state cultures enabled Watson to conclude that the reduced growth rate is caused by an increased requirement for glucose, the energy-yielding substrate, and that this is related to a higher demand for maintenance energy. Watson's data are important in providing a clear demonstration of one of the functions of maintenance energy in microorganisms; the increased requirement could be related to the maintenance of an intracellular concentration of sodium ten times lower than that of the extracellular medium.

\section{EARTH SCIENCE \\ Free Oscillations}

from our Geophysics Correspondent A RECENT article by V. S. Tuman (Nature, 229, 618; 1971) looks set fair to cause a major controversy in geophysics. First reports suggest that there are some geophysicists who are impressed with a spectacular achievement alongside those who believe the interpretation of the data to be utterly erroneous.

For many years, seismologists have hoped for a seismometer sufficiently quiet at long periods (up to one hour) to permit continuous spectral analysis of long stretches of record revealing the Earth's free oscillations. The Earth under the influence of some disturbance like an earthquake will be set into vibration in an infinite number of modes. The amplitude distribution of surface motion in such modes is described by spherical harmonics (although the pole of rotation will not coincide with the coordinate pole for the harmonics). Interaction between the free oscillations and the Earth's rotation removes degeneracy in spherical harmonics of the same degree, as does a non-spherically symmetric distribution of elastic constants and density. These effects are well documented theoretically and the splitting of spectral lines by rotation is well documented observationally. In addition to fundamental modes, there are a whole realm of overtones corresponding to spherical nodal surfaces within the Earth. Free oscillations of the Earth have a very close parallel in many quantum mechanical phenomena.

Calculation of the eigenfrequencies of standard Earth models is time consuming but not overwhelmingly difficult. Splitting of modes, for whatever cause, is also calculable. The gravest mode has a period of 53 minutes. Higher degree harmonics have shorter eigenperiods - the fortieth degree harmonic has a period of three and a half minutes. Overtones increase the density of eigenfrequencies at the shorter period end and splitting produces discernibly separate spectral peaks

\title{
Antigens and the Lymphoid System of Chimaeras
}

IT is now a quarter of a century since Owen's realization that some nonidentical twin pairs in cattle were chimaeras in the sense that each contained some red blood cells which had the antigenic phenotype of their twin. The discovery of this phenomenon influenced the development of the theory of immunological tolerance as a means by which an organism could learn not to react against antigenic components of itself. In the cattle twins it was supposed that in the early stages of foetal development the condition of synchory (placental fusion, essentially) had allowed the exchange of precursor cells between the twin foetuses and that these cells had been tolerated and accepted as self. But, aside from this corollary, the recognition of the chimaeric state established the principle that two different cell lives could grow in the same host animal and their products could be recognized and quantitated.

In 1956 it became clear that it was possible to produce chimaeras artificially when irradiated animals were injected with haematopoietic cells. This further discovery intensified the search for means of analysis of chimaeric conditions by the development of methods by which somatic cells could be recognized by other criteria than simple morphology. A variety of genetic, biochemical, functional and antigenic markers has emerged, the utilization of which has become a major pastime with cellular immunologists. Many experiments consist of the identification of cells in particular organs or responding to antigenic stimulation in chimaeric animals. In next Wednesday's Nature New Biology, Miller and Sprent comment on the use of a popular marker of thymus derived cells, the theta $(\theta)$ antigen.

Most cells in the thymus are strongly $\theta$ positive and it has reasonably been assumed the lymphocytes external to the thymus which are $\theta$ positive are of thymic origin-T cells. On the basis of this assumption Raff and his colleagues (Nature, 224, 378 ; 1969) have determined the numbers of $\mathrm{T}$ cells in the various lymphoid organs of a mouse. This analysis was not performed on chimaeras. Miller and Sprent have concentrated their attention on cells in the thoracic duct lymph of chimaeric mice and, like Raff and Wortis (Immunology, 18, 931 ; 1970), have concluded that they are mostly $\mathrm{T}$ cells. Miller and Sprent, however, took the matter further. They used an additional isoantigenic marker on transferred $T$ cell populations and found a discrepancy between the numbers of cells scored as $T$ cells in the secondary hosts, on the basis of $\theta$ antigenicity, and the numbers estimated by lysis with an isoantibody. Their conclusion is that at least some $T$ cells are efficient in $\theta$ antigenicity.

Some caution must therefore be exercised in experiments which are anti- $\theta$ antisera either as a means of quantitation or elimination of $\mathbf{T}$ cells. But, in spite of this setback, it is to be expected that the use of tissue specific surface antigens to analyse the structure of the lymphoid system is here to stay. 